\title{
Disruption of leptin receptor expression in the pancreas directly affects $\beta$ cell growth and function in mice
}

\author{
Tomoaki Morioka, ${ }^{1}$ Esra Asilmaz, ${ }^{2}$ Jiang Hu, ${ }^{1}$ John F. Dishinger, ${ }^{3}$ Amarnath J. Kurpad, ${ }^{1}$ \\ Carol F. Elias, ${ }^{4,5}$ Hui Li, ${ }^{1}$ Joel K. Elmquist, ${ }^{4}$ Robert T. Kennedy, ${ }^{3}$ and Rohit N. Kulkarni ${ }^{1}$
}

${ }^{1}$ Research Division, Joslin Diabetes Center, Boston, Massachusetts, USA. 2Laboratory of Molecular Genetics, The Rockefeller University, New York, New York, USA. ${ }^{3}$ Departments of Chemistry and Pharmacology, University of Michigan, Ann Arbor, Michigan, USA. ${ }^{4}$ Center for Hypothalamic Research and Division of Endocrinology and Metabolism, Department of Internal Medicine, University of Texas Southwestern Medical Center, Dallas, Texas, USA. Institute of Biomedical Sciences, University of Sao Paulo, Sao Paulo, Brazil.

\begin{abstract}
Obesity is characterized by hyperinsulinemia, hyperleptinemia, and an increase in islet volume. While the mechanisms that hasten the onset of diabetes in obese individuals are not known, it is possible that the adipose-derived hormone leptin plays a role. In addition to its central actions, leptin exerts biological effects by acting in peripheral tissues including the endocrine pancreas. To explore the impact of disrupting leptin signaling in the pancreas on $\beta$ cell growth and/or function, we created pancreas-specific leptin receptor (ObR) KOs using mice expressing Cre recombinase under the control of the pancreatic and duodenal homeobox 1 $(P d x 1)$ promoter. The KOs exhibited improved glucose tolerance due to enhanced early-phase insulin secretion, and a greater $\beta$ cell mass secondary to increased $\beta$ cell size and enhanced expression and phosphorylation of p70S6K. Similar effects on P70S6K were observed in MIN6 $\beta$ cells with knockdown of the ObR gene, suggesting crosstalk between leptin and insulin signaling pathways. Surprisingly, challenging the KOs with a high-fat diet led to attenuated acute insulin secretory response to glucose, poor compensatory islet growth, and glucose intolerance. Together, these data provide direct genetic evidence, from a unique mouse model lacking ObRs only in the pancreas, for a critical role for leptin signaling in islet biology and suggest that altered leptin action in islets is one factor that contributes to obesity-associated diabetes.
\end{abstract}

\section{Introduction}

The factors that promote $\beta$ cell failure and increase the incidence of diabetes in obese individuals are not fully understood. The presence of hyperglycemia and hyperphagia in obese individuals, despite the presence of high levels of circulating insulin and leptin, suggests these individuals are resistant to the actions of both hormones (1). The hypothalamic actions of leptin are relatively well characterized; however, the expression of the long form of the leptin receptor $(\mathrm{ObRb})$ in peripheral tissues, including the endocrine pancreas, indicates that leptin can also exert peripheral actions independent of its effects in the hypothalamus (2). For example, in vitro studies have reported inhibitory effects of leptin on insulin gene expression and insulin secretion in $\beta$ cell lines and isolated murine and human islets (2-4). Furthermore, leptin treatment of $o b / o b$ mice reversed hyperinsulinemia (5). Although the $d b / d b$ mouse, which carries a mutation in the ObR gene (6), manifests hyperinsulinemia and hyperplastic islets (7), it is unclear whether the alterations in islet growth and function in $d b / d b$ mice are due to a lack of direct leptin action in $\beta$ cells or secondary to the effects of insulin resistance in peripheral tissues.

To directly assess the role of leptin signaling in the pancreas, we used the Cre-loxP technique to create a mouse model that is

Nonstandard abbreviations used: Glut-2, glucose transporter-2; HFD, high-fat diet; ITT, insulin tolerance test; ObR, leptin receptor; ObRb, long form of the leptin receptor; Pdx1, pancreatic and duodenal homeobox 1; PTEN, phosphatase and tensin homolog.

Conflict of interest: The authors have declared that no conflict of interest exists. Citation for this article: J. Clin. Invest. 117:2860-2868 (2007). doi:10.1172/JCI30910 deficient in ObRs only in the pancreas. We report that the expression of Cre using the $P d x 1$ (pancreatic and duodenal homeobox 1) promoter results in efficient recombination of a loxP-containing ObR gene in the pancreas. Mice lacking ObRs in the pancreas (pancreas-ObR-KO) and fed normal chow manifested improved glucose tolerance, enhanced first-phase insulin response to glucose, and islet hyperplasia.

We also report upregulation in the islets of expression and phosphorylation of proteins in the insulin/IGF-I signaling pathway that are known to be important for the maintenance of islet growth and function (8-19). Qualitatively similar signaling data were observed in MIN6 $\beta$ cells with a knockdown of ObR using siRNA. Challenging the mice with a high-fat diet (HFD), however, led to significantly greater glucose intolerance in the KOs compared with controls. Together, these data provide genetic evidence for cross-talk between leptin and insulin signaling pathways in the regulation of islet biology, with implications for $\beta$ cell dysfunction in obesity.

\section{Results}

Creation of the pancreas-specific ObR-KO mice. We created a mouse with a pancreas-specific KO of $o b r$ by breeding mice carrying $o b r$ in which exon 1 was flanked with loxP sites (obrlox/lox; ObRlox) (20) with mice expressing cre driven by the $P d x 1$ promoter (21).

Both control (ObRlox) and $\mathrm{KO}$ mice were born at the expected Mendelian frequency, survived to adulthood, and were fertile. We performed RT-PCR analysis to evaluate efficiency of recombination of the ObRlox allele in the pancreas triggered by Cre expression. Expression of the gene coding for ObR was virtually absent in 

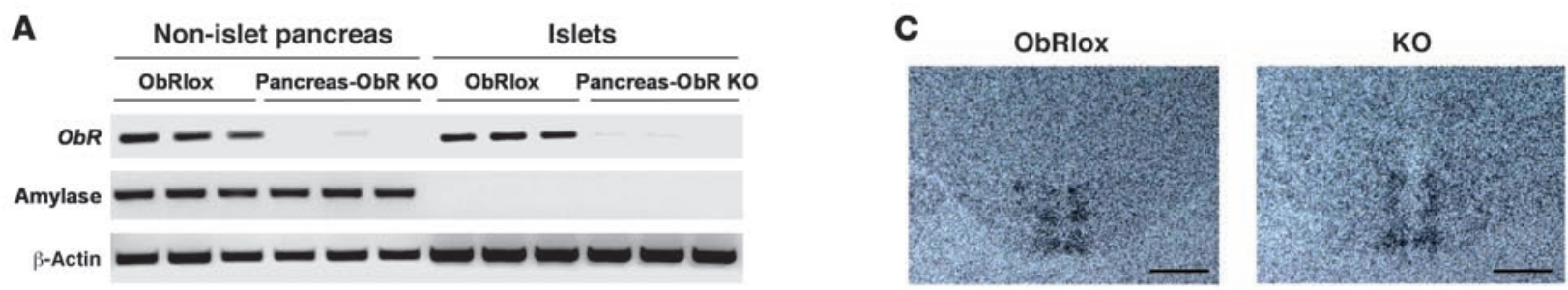

\section{B}

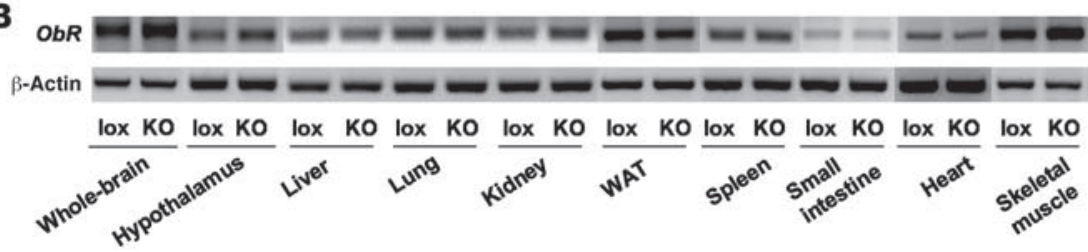

D
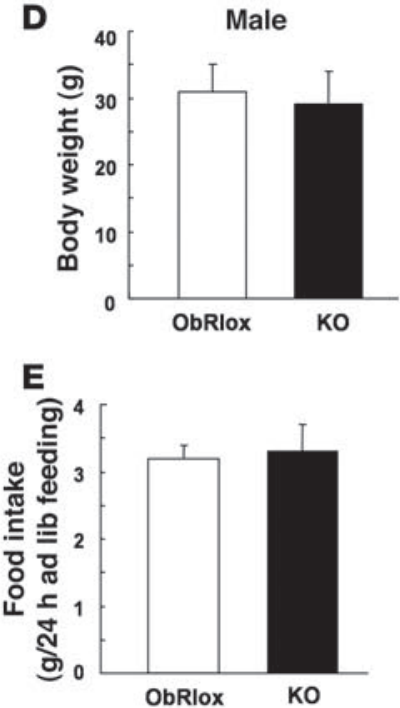
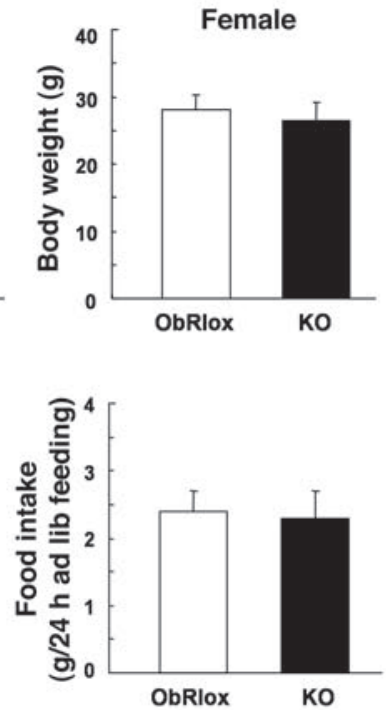

$\mathbf{F}$

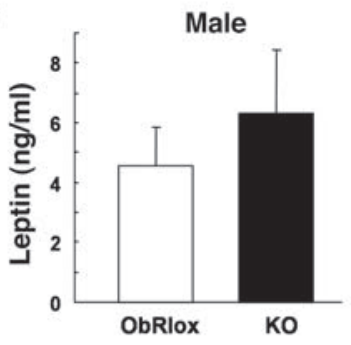

G

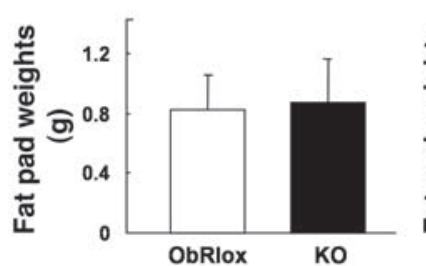

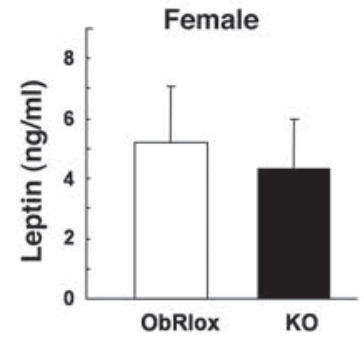

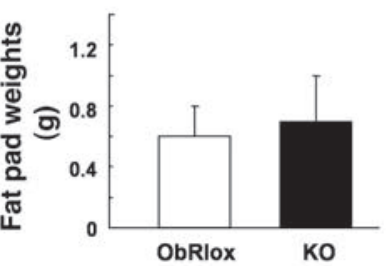

Figure 1

Evidence for deletion of ObR and physiological effects of pancreas-specific disruption of the ObR in pancreas-ObR-KO mice. (A) Representative data of expression of ObR gene in islets and non-islet pancreatic tissue in 6-month-old ObRlox and KO mice, as assessed by RT-PCR. Amylase was used as a marker for non-islet pancreas samples and $\beta$-actin as an internal control. $n=3$. (B) Representative data of expression of ObR gene assessed by RT-PCR in whole-brain extract, hypothalamus, liver, lung, kidney, white adipose tissue (WAT), spleen, small intestine, heart, and skeletal muscle in 6-month-old ObRlox (lox) and KO mice. $n=3$. (C) In situ hybridization for ObRb showing that it is localized in hypothalamus of both ObRlox and KO mice. Body weight (D), 24-hour ad libitum, food intake (E), fasting serum leptin concentration (F), and perigonadal fat pad weights $(\mathbf{G})$ in 4-month-old male and female ObRlox and KO mice. $n=4-6$; all $P=$ NS. Data are shown as mean \pm SEM. Scale bars: $500 \mu \mathrm{m}$.

the pancreas and islets but unaffected in extrapancreatic tissues in the KOs, indicating pancreas-specific recombination (Figure 1, A and B). The mRNA for ObRb assessed by in situ hybridization was expressed equally in the hypothalamus in both ObRlox and $\mathrm{KO}$ mice and confirmed the specificity of recombination (Figure 1C). In all cases, $\mathrm{KO}$ mice were compared with ObRlox controls.

Similar body weights, food intake, and fat mass in ObRlox control and KO mice. We next evaluated the physiological actions of leptin in the hypothalamus. Body weight, food intake, serum leptin concentrations, and perigonadal fat pad weights of 4-month-old male and female $\mathrm{KO}$ mice were similar to those of age- and sex-matched ObRlox controls (Figure 1, D-G). These findings confirm that ObRs in hypothalamus were functional in $\mathrm{KO}$ mice and that $\mathrm{KO}$ of ObRs in the pancreas did not modify body weight, food intake, fat mass, or circulating leptin levels in 4-month-old mutant animals.
Enhanced early-phase insulin secretion and improved glucose tolerance in $\mathrm{KO}$ mice. Leptin signaling suppresses insulin secretion (2-4). To evaluate the effects of pancreas-specific ObR deletion on $\beta$ cell secretory function in vivo, we measured acute insulin release in response to glucose stimulation. In male and female ObRlox controls, a significant increase in insulin secretion was observed 2 minutes following i.p. glucose injection, consistent with an acutephase insulin secretory response (Figure 2A). Interestingly, in KO mice, the acute-phase insulin secretory response to glucose was enhanced by 2 -fold in males and 3 -fold in females compared with controls (Figure 2A). Consequently, upon i.p. glucose challenge, $\mathrm{KO}$ mice showed a significantly lower glucose excursion, consistent with improved glucose tolerance (Figure 2B). Fasting circulating insulin levels in $\mathrm{KO}$ mice were approximately 2-fold higher than those in controls; the difference was statistically significant 


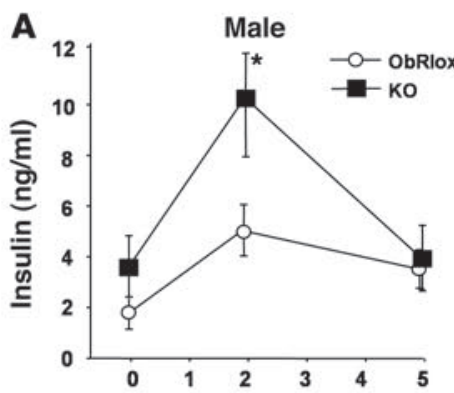

Min after i.p. glucose injection

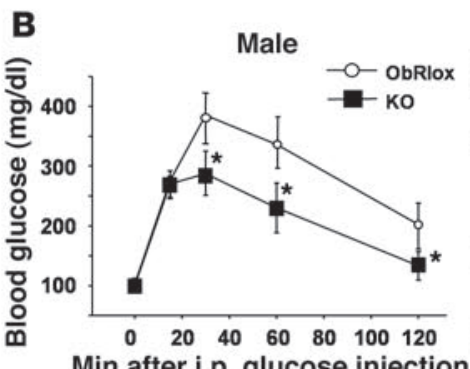

Min after i.p. glucose injection

E

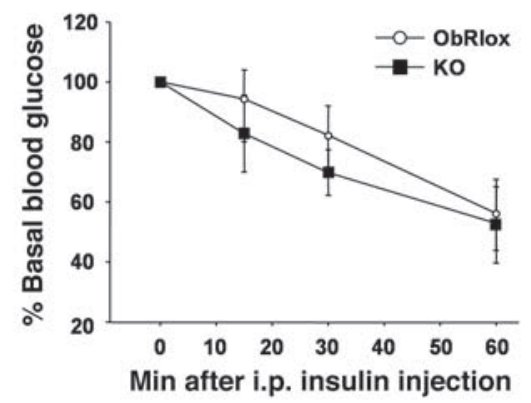

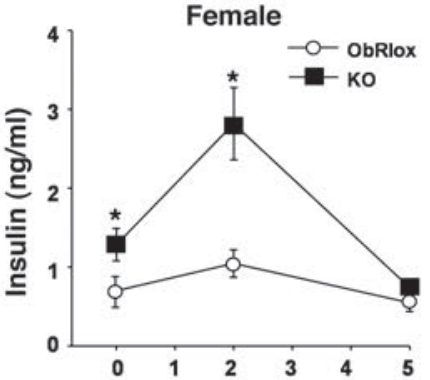

Min after i.p. glucose injection

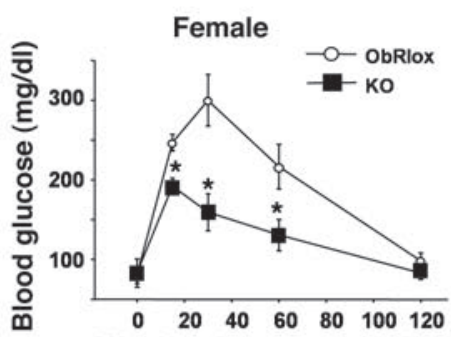

Min after i.p. glucose injection

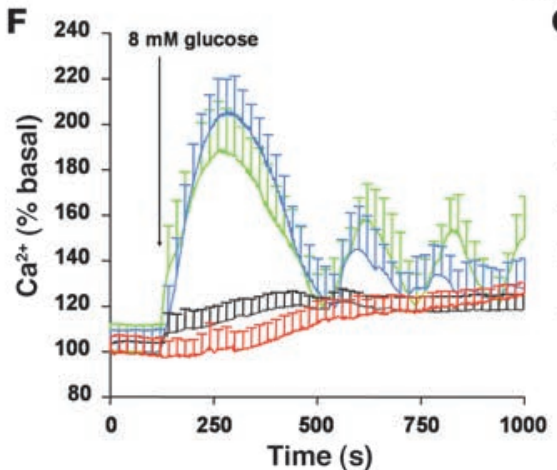

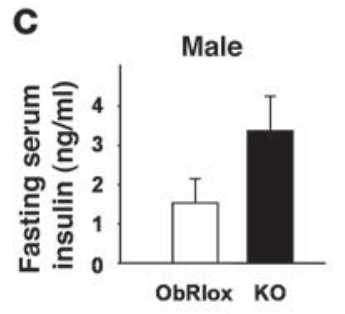
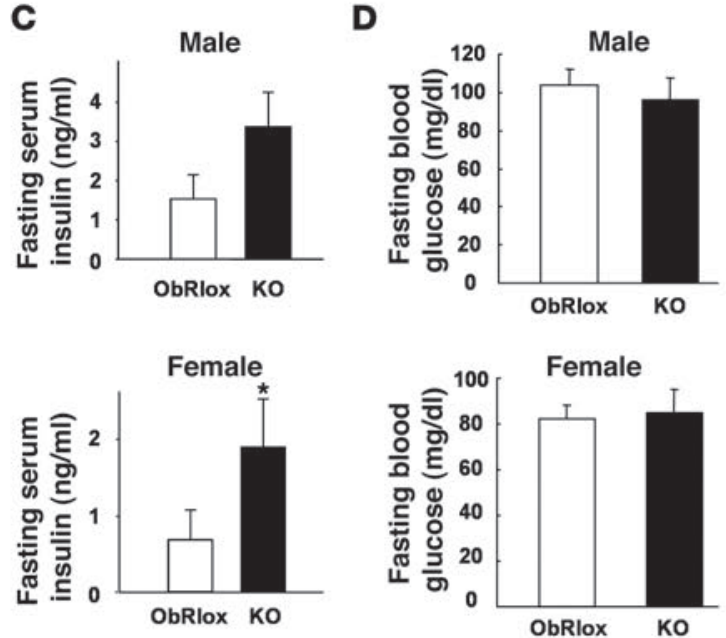

Figure 2

Improved glucose tolerance and enhanced early-phase insulin secretion in pancreas-ObR-KO mice. (A) Plasma insulin levels after an i.p. injection of glucose ( $3 \mathrm{~g} / \mathrm{kg}$ body weight) to evaluate acute-phase insulin secretion in 6 -month-old ObRlox and $\mathrm{KO}$ mice. ${ }^{*} P<0.05$ versus ObRlox controls. $n=6$. (B) Blood glucose after i.p. injection of glucose (2 g/kg body weight) in 6-month-old male and female ObRlox and KO mice. ${ }^{*} P<0.05$ versus ObRlox controls; $n=6-7$. Plasma insulin (C) and blood glucose (D) levels after overnight fasting in 6-month old male and female ObRlox and KO mice. ${ }^{*} P<0.05$ versus ObRlox controls; $n=6$. (E) Percent change in blood glucose after i.p. injection of insulin ( $1 \mathrm{U} / \mathrm{kg}$ body weight) in 6-month-old male ObRlox and KO mice. $n=7 ; P=\mathrm{NS}$. Data are shown as mean \pm SEM. Representative traces of intracellular $\mathrm{Ca}^{2+}$ flux $(\mathbf{F})$ and insulin secretion $(\mathbf{G})$ measured in primary size-matched islets isolated from 6-month-old male ObRlox and $\mathrm{KO}$ mice with or without $100 \mathrm{nM}(\mathbf{F})$ or $10 \mathrm{nM}(\mathbf{G})$ leptin. For area under the curve (AUC), $P<0.05$, ObRlox versus KO mice in each case; $P<0.05$, vehicle versus leptin in ObRlox mice for both $\mathrm{Ca}^{2+}$ and insulin; $P=$ NS for vehicle versus leptin+ in $\mathrm{KO}$ mice for both $\mathrm{Ca}^{2+}$ and insulin. $n=6$ islets from 3 individual mice in each group.

in females, and levels tended to be higher in males $(P=0.07)$, indicating increased basal insulin secretion (Figure 2C). Higher circulating levels of insulin were also observed in the random-fed state in $\mathrm{KO}$ mice compared with controls (male: $4.7 \pm 0.3$ versus $2.3 \pm 0.4$ $\mathrm{ng} / \mathrm{ml}, P<0.05, n=5$; female: $3.6 \pm 0.4$ versus $1.9 \pm 0.3 \mathrm{ng} / \mathrm{ml}$, $P<0.05, n=4-5)$. Blood glucose levels in $\mathrm{KO}$ mice did not differ from those in ObRlox mice in the fasting state (Figure 1D) and tended to be lower, although the difference was not statistically significant in random-fed states (male: KO, $102 \pm 14$ versus ObRlox, $110 \pm 18 \mathrm{mg} / \mathrm{dl}$, $n=5$; female: $\mathrm{KO}, 114 \pm 16$ versus ObRlox, $131 \pm 20 \mathrm{mg} / \mathrm{dl}, n=5$ ). Whole-body insulin sensitivity evaluated by i.p. insulin tolerance tests (ITTs; 1 U Humulin/kg body weight) showed no differences between the groups (Figure 2E). Fasting plasma glucagon levels did not show significant differences between groups (male: ObRlox, $66 \pm 12$ versus KO, $52 \pm 14 \mathrm{pg} / \mathrm{ml}$; female: ObRlox, $58 \pm 14$ versus $\mathrm{KO} 64 \pm 16 \mathrm{pg} / \mathrm{ml}, n=5 ; P=\mathrm{NS})$.

To evaluate insulin secretion in vitro, we cultured freshly isolated islets for 48 hours in glucose at physiological concentrations and then subjected single, size-matched islets from ObRlox or KO mice to step-up glucose stimulation. We observed significantly enhanced $(P<0.05) \mathrm{Ca}^{2+}$ flux and insulin secretion in response to an increase in glucose concentration from 3 to $8 \mathrm{mM}$ in the KOs (Figure 2, F and G). Further, consistent with previous reports $(2,3)$, leptin treatment suppressed $\mathrm{Ca}^{2+}$ flux and insulin secretion in control islets, while no effects were evident in the $\mathrm{KO}$ islets. These results suggest that absence of leptin signaling 
A

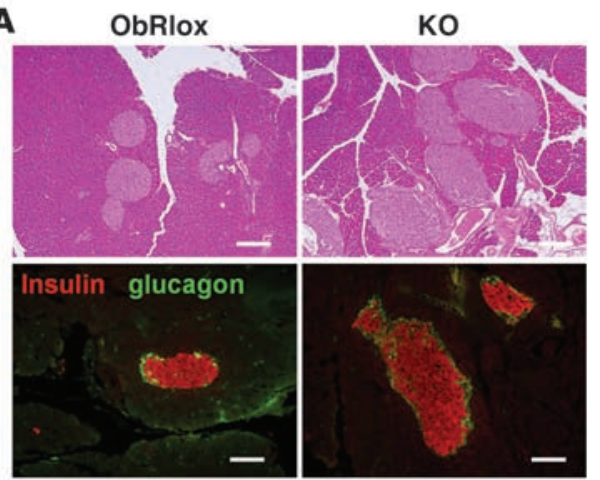

C

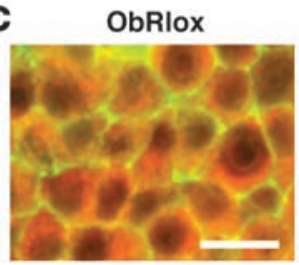

Insulin

KO

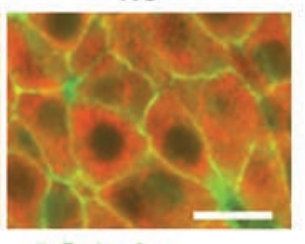

$\beta$-Catenin
B
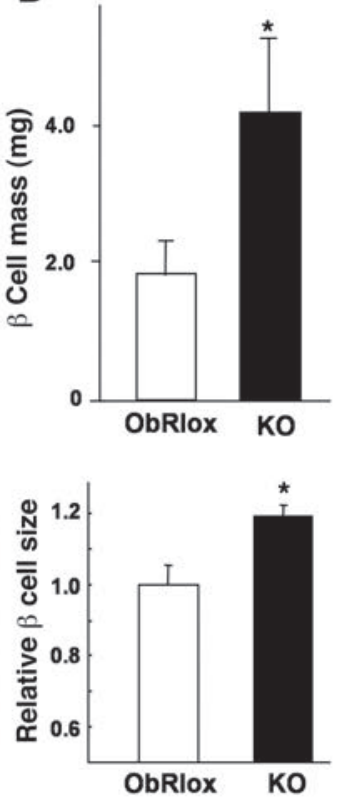

E

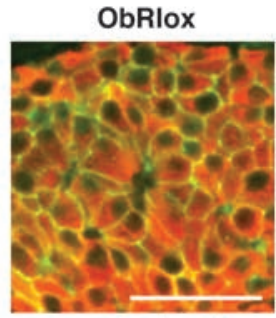

Insulin

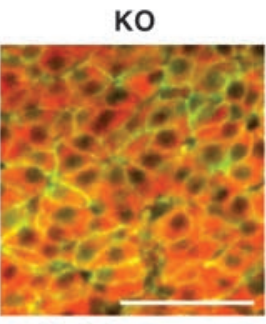

Glut-2
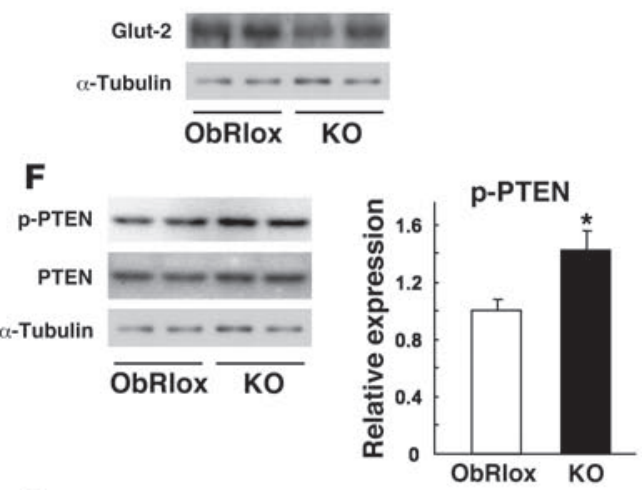

G

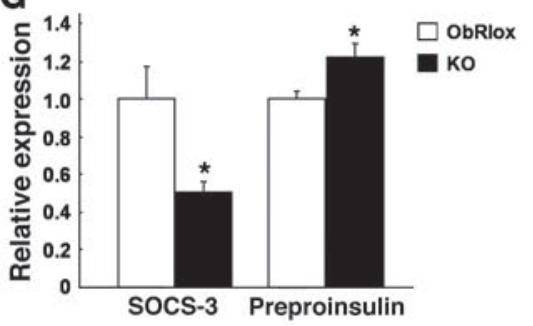

D

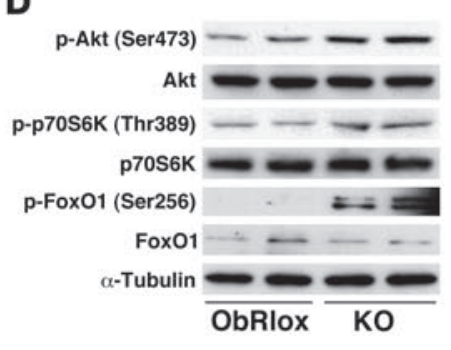

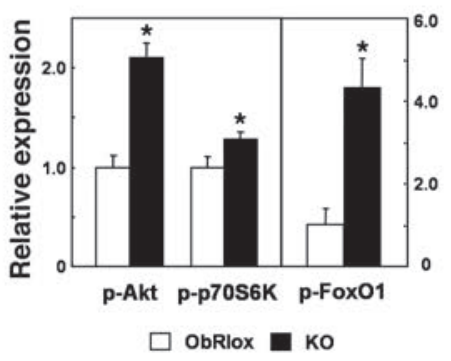

Figure 3

Increase in islet size and $\beta$ cell mass and enhanced expression of insulin signaling proteins in islets of pancreas-ObR-KO mice. (A) H\&E staining or immunofluorescence staining for insulin and glucagon in pancreas sections of ObRlox and KO mice. Scale bars: $100 \mu \mathrm{m}$. (B) $\beta$ Cell mass estimated by morphometric analysis. ${ }^{*} P<0.05$ versus ObRlox; $n=4$. (C) Immunofluorescence staining for insulin and $\beta$-catenin in pancreas sections to determine $\beta$ cell size. Relative $\beta$ cell size (mean from $n \geq 200$ cells counted) is shown in the graph. ${ }^{*} P<0.05$ versus ObRlox; mean \pm SEM, $n=3$. Scale bars: $10 \mu \mathrm{m}$. (D) Western blots of total islet lysates for p-Akt (Ser473), Akt, p-p70S6K (Thr389), p70S6K, p-FoxO1 (Ser256), FoxO1, and $\alpha$-tubulin as a loading control. The relative expression of p-Akt, p-p70S6K, and p-FoxO1 normalized to each total protein is shown in the graph. ${ }^{\star} P<0.05$ versus ObRlox; $n=4-6$. (E) Immunofluorescence for Glut-2 and insulin in pancreas sections of ObRlox and KO mice. Scale bars: $50 \mu \mathrm{m}$. Western blots of total islet lysates for Glut-2 (E, lower panel), p-PTEN, and PTEN (F), normalized to $\alpha$-tubulin. The relative expression of p-PTEN normalized to $\alpha$-tubulin is shown in the graph. ${ }^{*} P<0.05$ versus ObRlox; $n=4$. (G) Expression of SOCS-3 and preproinsulin in $\mathrm{ObRlox}$ and $\mathrm{KO}$ islets assessed by quantitative real-time PCR. ${ }^{*} P<0.05$ versus ObRlox; $n=4-6$. Data were obtained from pancreas or islet samples from 4- to 6-month-old mice and are shown as mean \pm SEM.

allows enhanced insulin secretion from cultured islets, consistent with the observations in vivo.

Islet byperplasia and increased expression of insulin signaling proteins in $\mathrm{KO}$ islets. In addition to growth factors, leptin signaling has also been implicated in $\beta$ cell growth in vitro (22). To evaluate whether disruption of ObRs in the pancreas affects islet morphology and $\beta$ cell growth in vivo, we performed immunohistochemical analyses of pancreas sections using a cocktail of antibodies to non- $\beta$ cell hormones $(23,24)$. We observed a 2 -fold increase in $\beta$ cell mass in $\mathrm{KO}$ mice that was secondary to an increase in $\beta$ cell size (Figure 3, A-C) but not due to enhanced mitosis (control, $0.27 \% \pm 0.2 \%$ versus $\mathrm{KO}, 0.37 \% \pm 0.2 \% \mathrm{BrdU}^{+} \beta$ cells [total 500 $\beta$ cells counted], $n=4 ; P=\mathrm{NS}$ ). We did not observe differences in expression of cleaved caspase-3, suggesting that the increase in $\beta$ cell mass observed in KO mice is unlikely due to altered $\beta$ cell apoptosis (data not shown).

To explore the pathways that mediate an increase in islet hyperplasia, we considered previous reports that proteins in the insulin/ IGF-I pathways regulate $\beta$ cell size and survival (8-19). Western blotting of islet lysates showed significantly enhanced phosphorylation of PKB/Akt at Ser473 (p-Akt), p70 S6 kinase at Thr389 (p-p70S6K), and FoxO1 at Ser256 (p-FoxO1) in KO mice by 2.1-, 1.3-, and 4.3-fold, respectively, compared with controls, with unaltered expression of total Akt, p70S6K, or FoxO1 protein levels (Figure 3D). These results suggest that, in the absence of leptin signaling, the increase in phosphorylation of $\mathrm{P} 70 \mathrm{~S} 6 \mathrm{~K}$ and $\mathrm{PKB} / \mathrm{Akt}$, which are important for determining $\beta$ cell size and survival, respectively $(16-18)$, likely contributes to an increase in $\beta$ cell size and islet mass in pancreas-ObR-KO mice. 


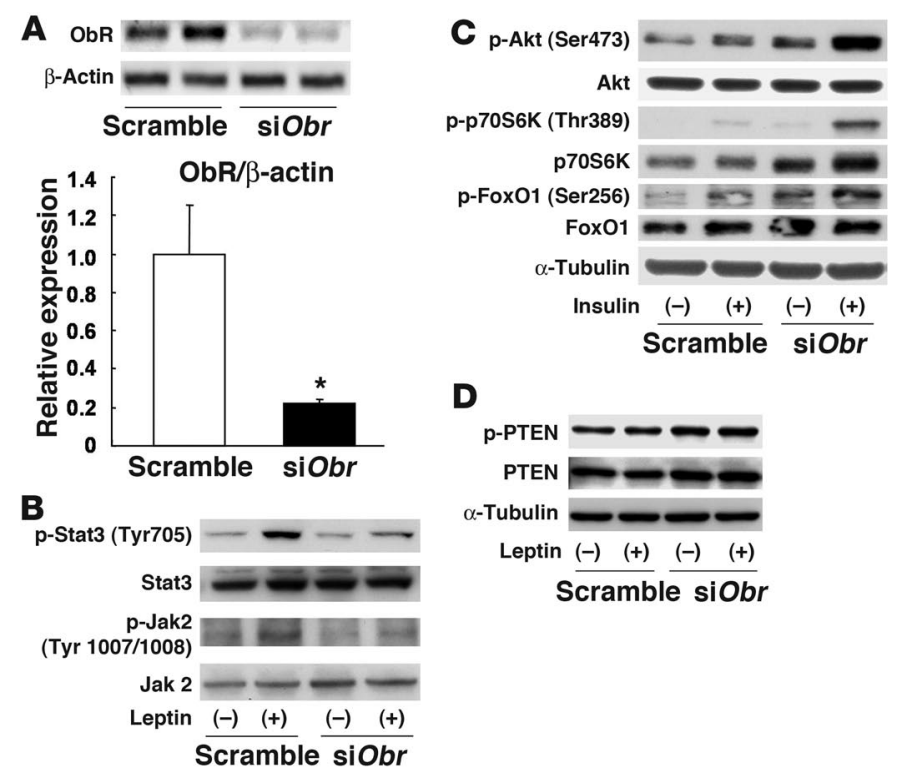

\section{Figure 4}

Increased expression and phosphorylation of insulin-signaling proteins in MIN6 $\beta$ cells with knockdown of ObR gene. (A) RTPCR analysis of ObR and $\beta$-actin in MIN6 $\beta$ cells transfected with scrambled control or siRNA for ObR $(\mathrm{siObR})$ for 48 hours. The relative expression of $\mathrm{ObR}$ analyzed by real-time PCR was normalized to $\beta$-actin. ${ }^{*} P<0.01$ versus scrambled control; mean \pm SEM; $n=6$. (B) MIN6 $\beta$ cells were transfected with scrambled control or siObR for 48 hours and treated with or without $10 \mathrm{nM}$ leptin for 15 minutes following overnight serum starvation. Total cell lysates were extracted and subjected to Western blot analysis for $\mathrm{p}$-Stat3, Stat3, p-Jak2, and Jak2. Each experiment was performed at least 3 times. (C and D) Western blot analyses for p-Akt (Ser473), Akt, p-p70S6K and p70S6K, p-FoxO1 (Ser256), FoxO1 (C), p-PTEN, and PTEN (D) normalized to $\alpha$-tubulin protein in total cell lysates of MIN6 $\beta$ cells transfected with scrambled control or siObR and treated with or without $100 \mathrm{nM}$ insulin (C) or $10 \mathrm{nM}$ leptin (D) for 15 minutes following overnight serum starvation. Each experiment was performed at least 3 times.
To further explore the possible mechanisms underlying the increased insulin-secretory function with enhanced insulin receptor signaling pathway in $\mathrm{KO}$ mouse islets, we evaluated the expression of glucose transporter-2 (Glut-2) in $\beta$ cells. Immunofluorescence analyses showed similar expression patterns of Glut-2 in both groups (Figure 3E, upper panel). Further, Western blotting of islet lysates showed the tendency of Glut-2 to decrease in KOs, but the difference was not statistically significant (Figure 3E, lower panel), suggesting that alterations in Glut-2 expression are unlikely to contribute substantially to the higher insulin secretion in the KOs.

Since phosphatase and tensin homolog (PTEN), a major negative regulator of the PI3K/Akt signaling pathway, was recently reported to be regulated by leptin in hypothalamic cell lines and pancreatic $\beta$ cells (25), we evaluated phosphatase activity and protein content in $\mathrm{KO}$ islets. By Western blotting, we observed a mild but significant upregulation of PTEN phosphorylation ( $\mathrm{p}$-PTEN) in the KOs compared with controls (Figure 3F), while no differences were observed in total PTEN protein expression $(\mathrm{KO}, 122 \% \pm 14 \%$ of control; $n=4 ; P=\mathrm{NS}$ ). These data are compatible with enhanced $\mathrm{PI} 3 \mathrm{~K} /$ Akt signaling in the KOs, in part due to the attenuation of PTEN, a negative regulator of insulin signaling.

SOCS-3 also acts as a negative regulator of insulin signaling and has been reported to link cytokine signaling with insulin resistance (26). In $\beta$ cells, SOCS-3 has been reported to be induced by leptin and inhibit preproinsulin gene expression (27). Consistent with these observations, we observed a significant decrease in the expression of SOCS-3 by quantitative real-time PCR in KO islets that was associated with a mild but significant increase in preproinsulin gene expression (Figure 3G). These results confirm the link between SOCS-3 and insulin gene expression and provide additional evidence for a regulatory role for leptin/insulin signaling in $\beta$ cell secretory function.

Enhanced insulin signaling in MIN6 $\beta$ cells with ObR gene knockdown. To further evaluate the potential interactions between insulin and leptin signaling pathways in vitro, we utilized MIN6 $\beta$ cells with ObR gene knockdown using siRNA. Transfection of siRNA oligonucleotides for mouse ObR ( $\mathrm{siObr}$ ) resulted in an approximately $80 \%$ reduction in $O b R$ gene expression in MIN6 $\beta$ cells and was confirmed by quantitative real-time PCR (Figure 4A). In control MIN6 $\beta$ cells transfected with scrambled RNA, leptin treatment promoted tyrosine phosphorylation of Stat 3 (p-Stat3) and Jak2 (p-Jak2), while virtually no effect was observed in siObr-knockdown cells (Figure 4B), indicating that leptin-stimulated Jak2/ Stat 3 signaling is attenuated in cells with $O b R$ knockdown.

Next, to assess alteration in proteins in the insulin-induced signaling pathways in MIN6 $\beta$ cells with ObR knockdown, we treated MIN6 $\beta$ cells with insulin after transfection of siObr or scrambled RNA. MIN6 $\beta$ cells with ObR knockdown showed an increase in protein expression of $\mathrm{p} 70 \mathrm{~S} 6 \mathrm{~K}$ and FoxO1, in the basal state, and elevated insulin-induced phosphorylation of PKB/Akt (Ser473), p70S6K (Thr389), and FoxO1 (Ser256) (Figure 4C). We also observed mildly elevated PTEN phosphorylation in MIN6 $\beta$ cells with $O b R$ knockdown in both basal and leptin-treated states (Figure 4D). These findings in MIN6 $\beta$ cells are in agreement with signaling data in islets from pancreas-ObR-KO mice (Figure 3, D and F) and suggest that the phenotypes in KOs are due to a primary defect in $\beta$ cells and unlikely due to altered leptin action in non$\beta$ cells, neural, or other circulating factors. Indeed, no significant differences were detected in circulating glucagon or somatostatin (data not shown) levels between groups.

Impaired glucose tolerance in pancreas-ObR-KO mice induced by HFD feeding. In addition to its role in islet function and survival, leptin modulates lipid metabolism in islets $(28,29)$. To explore whether the enhanced insulin secretion observed in the KOs on a normal chow diet protects the mutants in the face of caloric excess, we fed control and $\mathrm{KO}$ mice with an HFD or normal chow for 12 weeks. After high-fat feeding, as expected, mice in both groups were significantly heavier and exhibited insulin resistance compared with their littermates on normal chow diet, confirming the effects of diet-induced obesity (Figure 5, A and B). Further, the HFD-fed mice in the $\mathrm{KO}$ and control groups responded by gaining similar body weights (Figure 5A) and were equally insulin resistant (Figure $5 \mathrm{~B}$ ). Surprisingly, however, the KO mice on HFD exhibited impaired glucose tolerance that was, in part, due to blunted acute insulin response to glucose compared with the controls (Figure 5 , C-E). Furthermore, while control mice on HFD developed islet 
A

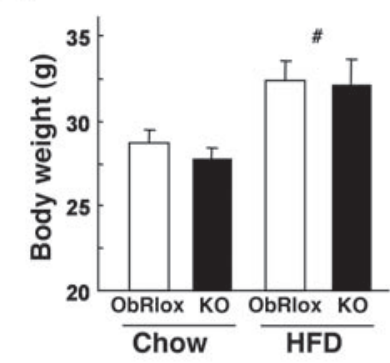

C

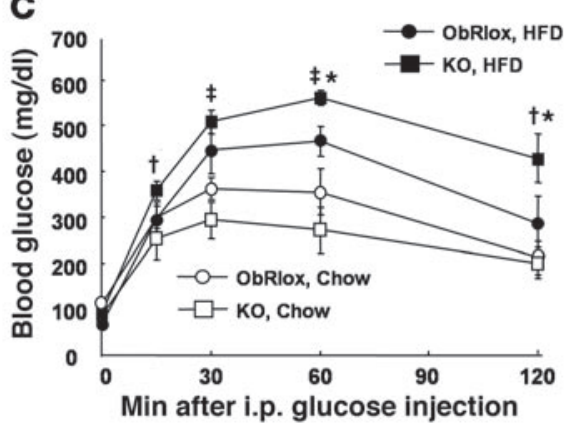

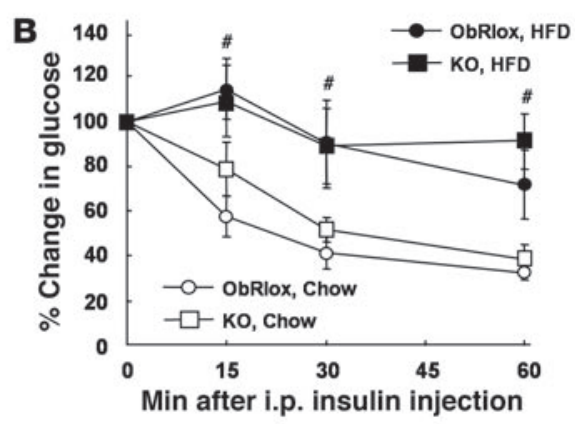

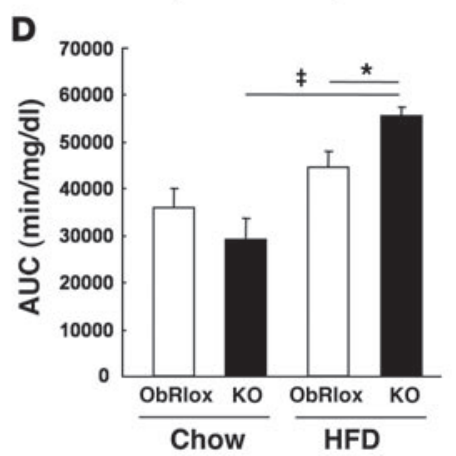

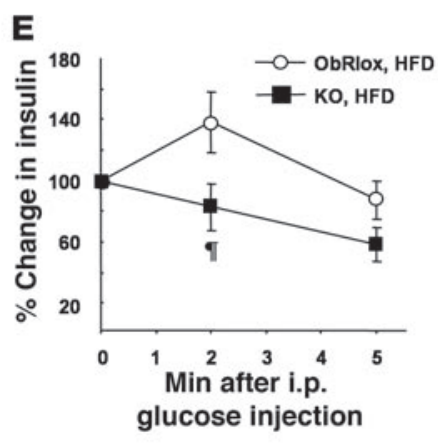

F ObRlox

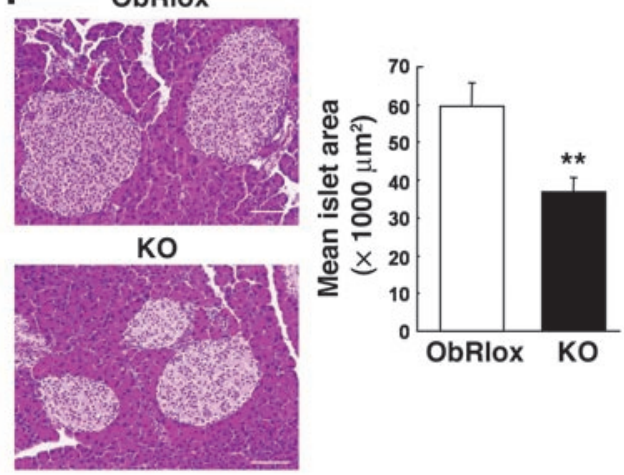

\section{Figure 5}

Diet-induced obesity leads to impaired glucose tolerance in pancreas-ObR-KO mice. Body weight (A), ITT results (B), glucose tolerance test (GTT) results (C), and area under the curve for the GTT results (D) in 4-month-old male ObRlox and $\mathrm{KO}$ mice on regular chow (Chow) or HFD for 12 weeks. ${ }^{\#} P<0.05$, HFD $(n=10)$ versus Chow $(n=6)$; ${ }^{*} P<0.05$, KO mice on HFD versus ObRlox mice on HFD, $n=5$; ${ }^{\top} P<0.05, \neq P<0.01$, $\mathrm{KO}$ mice on HFD versus KO mice on Chow, $n=5$ (KO) or 3 (Chow). (E) Percent change in plasma insulin levels after i.p. glucose injection $(3 \mathrm{~g} / \mathrm{kg}$ body weight) in ObRlox and KO mice on HFD for 12 weeks. $\urcorner P=0.07 ; n=4$. (F) Left: H\&E staining in representative pancreas sections of ObRlox and KO mice on HFD for 12 weeks. Scale bars: $100 \mu \mathrm{m}$. Right: Mean islet area from at least 10 islets from 5 individual mice for each genotype. ${ }^{\star \star} P<0.01$ versus ObRlox. Data are shown as mean \pm SEM. hyperplasia to compensate for insulin resistance compared with mice on normal chow, the $\mathrm{KO}$ mice showed a significantly reduced islet mass (61.8\% of control) after high-fat feeding (Figure $3 \mathrm{~A}$ and Figure 5F). Leptin has been reported to regulate lipid metabolism in $\beta$ cells and to protect islets from the effects of lipid overload $(28,30)$. Lipid accumulation in the islets, in turn, is known to impair islet secretory function and viability (28). Thus, it is possible that switching the diet from normal chow to high-fat in mice lacking leptin signaling in islets leads to lipid accumulation in the islets and consequent poor insulin secretory response and attenuated islet growth, leading to altered glucose homeostasis. Further molecular studies are necessary to dissect the pathways involved in leptin modulation of lipid metabolism and in the cross-talk with growth factor pathways regulating the $\beta$ cell proliferation response in these mutants in the context of an HFD.

\section{Discussion}

To directly assess the role of ObR signaling in the growth and function of pancreatic $\beta$ cells, we created a genetic model using a mouse expressing Cre-recombinase driven by the $P d x 1$ promoter for 2 reasons. First, the absence of Cre expression in the brain of the PdxCre mouse eliminates the possibility of recombination in the hypo- thalamus, which is rich in ObR-positive neurons (21). Second, this approach allowed us to examine the effect of recombination of the ObR gene around E8.5, when the $P d x 1$ promoter is switched on earlier than expression of the rat insulin promoter (21). Our studies provide genetic evidence for a direct role for leptin in the endocrine pancreas, in contrast to a recent report that used a mouse expressing Cre recombinase on the rat insulin promoter that is expressed later during development ( E9.5) and is also expressed in some neurons in the hypothalamus (31). Considering the dominant role of leptin in the hypothalamus, it is likely that the islet phenotype in mice generated with the rat insulin promoter-driven Cre is masked by alterations in hypothalamic function affecting insulin sensitivity and fat mass.

Previous studies have reported that leptin signaling interacts with proteins in the insulin-signaling pathway to exert its biological actions in several tissues. For example, leptin activates the IRS/ PI3K pathway in hypothalamic neurons to regulate food intake and body weight (1) and to stimulate glucose transport and glycogen synthesis in muscle cells (32). In hepatocytes, leptin activates IRS-associated PI3K and PDE3B to promote inhibition of cAMP elevation by glucagon $(33,34)$. In $\beta$ cell lines and isolated islets, leptin inhibits insulin secretion through PI3K-dependent PDE3B 


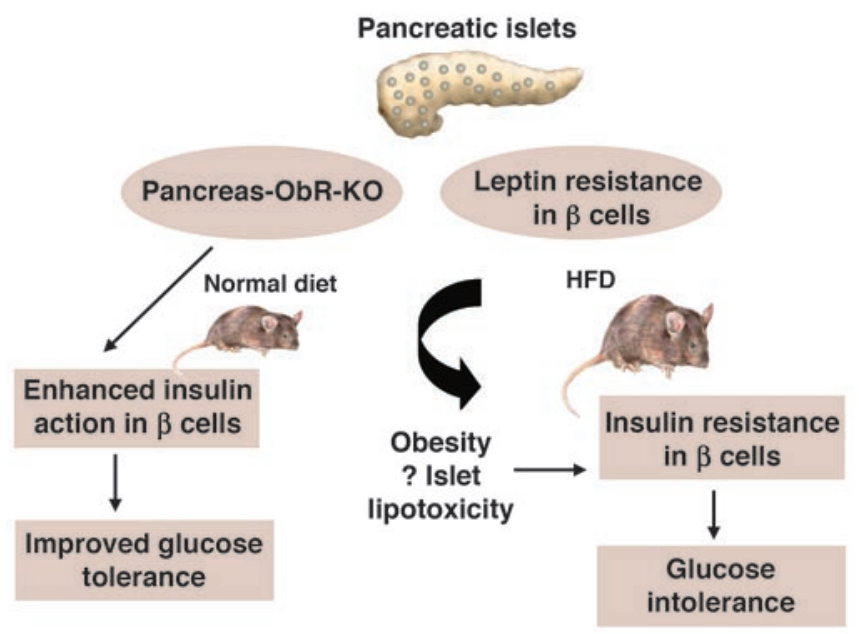

Figure 6

Schematic proposing a potential role for leptin signaling in islets in the development of obesity-associated diabetes.

activation or $\mathrm{K}_{\mathrm{ATP}}$ channel activation (2). Consistent with an inhibitory role for leptin, pancreas-ObR-KO mice showed enhanced acute-phase glucose-stimulated insulin secretion and elevated fasting plasma insulin concentrations. The lack of a corresponding significant decrease in fasting glucose levels suggests that pathways independent of leptin may play a role during the fasting state. Alternatively, the inhibitory effects of leptin may be more apparent when GLP-1 is secreted during the fed state (2). Furthermore, our studies provide evidence, for the first time to our knowledge, that absence of leptin action promotes $\beta$ cell growth by affecting the phosphorylation of p70S6K and Akt; the qualitatively similar signaling defects observed in MIN6 $\beta$ cells with an siRNA knockdown of ObRs suggest that the phenotype in mice in vivo is primarily due to an effect in $\beta$ cells. Indeed, simultaneous regulation of p70S6K and Akt has been reported to augment growth in other cell types (35). The phenotype in pancreas-ObR-KO mice are independent of direct effects in $\alpha$ cell function since ObRs are not expressed in $\alpha$ cells (2); however, pacarine effects of somatostatin due to altered $\delta$ cell function cannot be ruled out. Furthermore, the effects of ObR-KO in the potential modulation of exocrine pancreatic tissue needs further investigation. The findings in the pancreas-ObR$\mathrm{KO}$ mouse provide one explanation for the increase in $\beta$ cell mass observed at an age that precedes weight gain or hyperinsulinemia in Zucker fatty rats, which also lack leptin action (36). Similarly, the islet hyperplasia observed in mice bearing defects in the leptin $(o b / o b)$ or the ObR $(d b / d b)$ gene is likely due, in part, to deficient leptin signaling and consequent enhanced insulin action in $\beta$ cells $(7,37)$. Thus, it is important to consider the effects of lack of leptin signaling in islets in these widely used naturally occurring rodent models when studying glucose homeostasis.

The reduced SOCS-3 and mildly increased preproinsulin gene expression in the islets of $\mathrm{KO}$ mice are compatible with the reports that SOCS-3 is a leptin-induced negative regulator of leptin signaling (1) and an in vitro study showing inhibition of preproinsulin gene expression by leptin induction of SOCS-3 (27). Thus, the reduced expression of SOCS-3 in the KO islets is consistent with enhanced phosphorylation of PI3K/Akt signaling in islets lacking ObR expression (26). The reduced SOCS-3 expression might con- tribute, in part, to enhanced insulin secretion and islet hyperplasia by upregulating insulin gene expression and insulin signaling in islets lacking leptin signaling. Finally, the elevated PTEN phosphorylation in KO islets and MIN6 $\beta$ cells with ObR knockdown could indirectly enhance signaling via the PI3K/Akt pathway in the $\beta$ cells, since phosphorylation of PTEN leads to inactivation of its phosphatase activity. One interpretation of these studies is that absence of leptin signaling allows enhanced action of insulin on $\beta$ cells in an autocrine/paracrine manner to promote secretory function and/or growth.

To explore whether the effect of enhanced acute-phase secretion and improved glucose tolerance observed in the $\mathrm{KO}$ mice on normal chow persists and protects KOs against the effects of dietinduced obesity, we fed the KO mice with an HFD. Surprisingly, we observed glucose intolerance that was significantly worse in the KOs compared to that in the controls. Indeed, these findings bring into context the role of leptin in regulating lipid metabolism and islet function/growth $(28,29)$. For example, expression of wild-type ObRs in the islets of Zucker diabetic fatty rats attenuates accumulation of lipid content and rescues diabetogenic phenotypes such as impaired insulin secretion and $\beta$ cell apoptosis (28). Thus, the attenuated acute-phase secretory response and an inadequate compensatory islet growth response in the KOs on the HFD may be due to secondary effects of lipid overload in the islets as a consequence of absent leptin signaling and is one possible mechanism underlying the impaired glucose tolerance in the pancreas-ObR-KO mice (summarized in Figure 6). Alternatively, insulin resistance at the level of the $\beta$ cell itself in the KOs may directly contribute, in part, to a failure of the $\beta$ cell to compensate for HFD-induced insulin resistance. In any case, the specific interactions among leptin signaling, lipid metabolism, and growth factor proteins contributing to insulin resistance in $\beta$ cells in the context of obesity warrants further investigation.

A distinct difference between the pancreas-ObR-KO model reported in this article, created using $\mathrm{Pdx}$-Cre, and that reported by Covey et al, using RIP-Cre (31), is the recombination in the hypothalamus in the latter. Although the mice reported by Covey et al. did not manifest enhanced food intake, the increased body weight, fat mass, serum leptin levels, and plasma and liver triglyceride content, even when the mice were fed normal chow, together can directly contribute to glucose intolerance and poor insulin sensitivity in these mice $(20,38)$. In contrast, the pancreas-ObR-KOs on normal chow did not manifest alterations in insulin sensitivity but exhibited improved glucose tolerance due to enhanced insulin secretion that is consistent with a lack of tonic inhibitory action of leptin on $\beta$ cell secretion $(2,3)$. Furthermore, we also provide evidence for crosstalk between leptin and insulin signaling pathways that contributes to alterations in $\beta$ cell growth and function. Thus, the phenotype in pancreasObR-KO mice reflects the direct effects of absent leptin signaling in the islets, independent of the hypothalamus, and provides a straightforward model to study the role of leptin signaling in the development of obesity-associated diabetes. It will be especially instructive to explore whether disruption of both leptin and insulin signaling in the $\beta$ cells, akin to leptin and insulin resistance associated with diet-induced obesity, leads to a greater susceptibility to development of diabetes.

In summary, we have created a unique genetic model to directly demonstrate the consequences of lack of functional ObRs in islet $\beta$ cells. It is tempting to speculate that leptin resistance in pancreatic 
$\beta$ cells is one factor that contributes to the hyperinsulinemia, $\beta$ cell failure, and consequent glucose intolerance in the obese state.

\section{Methods}

Animals and genotyping. ObRlox mice were created by homologous recombination using the ObR gene with loxP sites flanking exon 1 , as previously described (20), and were maintained on a C57BL/6 background. Transgenic mice expressing Cre recombinase under the control of the mouse $P d x 1$ promoter (Pdx-Cre mice) (21) were maintained on a C57BL/6 background and bred with $\mathrm{Obr}$ floxed mice. All animals were housed in specific pathogen-free facilities and maintained on a 12-hour light/ 12-hour dark cycle and fed standard rodent chow at the Foster Animal Laboratory, Brandeis University, Waltham, Massachusetts, USA. We used both male and female mice for all experiments. All protocols for animal use were approved by the Institutional Animal Care and Use Committee of the Joslin Diabetes Center and Brandeis University and were in accordance with NIH guidelines.

For the high-fat feeding study, 6-week-old ObRlox control and pancreasObR-KO male mice were fed either an HFD or regular chow for 12 weeks as described previously (11). Body weights were followed weekly, and glucose tolerance tests (GTTs) and ITTs were performed during the last week of feeding as described below.

Genotyping was performed on DNA isolated from the tails of 3- to 4-weekold mice by PCR. The primers for the obrlox gene were $5^{\prime}$-GTCACCTAGGTTAATGTATTC-3' (forward) and 5'-TCTAGCCCTCCAGCACTGGAC-3' (reverse) and for the Cre transgene were 5'-TGCCACGACCAAGTGACAGC-3' (forward) and 5'-CCAGGTTACGGATATAGTTCATG-3' (reverse).

Physiological measurements. Food intake of the mice was measured as described previously (39). We determined blood glucose values from whole venous blood using an automated glucose monitor (Glucometer Elite; Bayer) and measured serum insulin and leptin levels by ELISA, using mouse insulin or leptin standards (Crystal Chem Inc.). GTTs and acute insulin secretion tests were performed on animals that had been fasted overnight for 16 hours $(8,9)$. For GTTs, glucose levels were measured from blood collected from the tail immediately before and 15, 30, 60, and 120 minutes after i.p. glucose injection. For acute insulin secretion tests, blood samples were collected before (time 0 ) and 2 and 5 minutes after i.p. glucose injection $(3 \mathrm{~g} / \mathrm{kg}$ body weight). ITTs were performed as previously described (9). Blood glucose levels were determined immediately before and 15,30 , and 60 minutes after injection of insulin at random-fed state (1 U/kg body weight) (Humulin R; Lilly).

$R T-P C R$ and quantitative real-time PCR. Islets were isolated from mice as described previously (3). Total RNA from islets, non-islet tissue, and whole-brain extract, hypothalamus, liver, lung, kidney, white adipose tissue, spleen, small intestine, heart, and skeletal muscle was extracted using RNeasy Mini Kit (QIAGEN). Expression of the obr gene in the different tissues was detected by RT-PCR $(8,9)$. The primers for mouse $o b r$ were $5^{\prime}$-TGATTTGTGTTTGATTTATTGTTT-3' (forward) and 5'TATTCGTAGACGTGGGTTGG-3' (reverse); for mouse $\beta$-actin were 5'-AGGGCTATGCTCTCCCTCAC-3' (forward) and 5'-AAGGAAGGCTGGAAAAGAGC-3' (reverse); and for mouse amylase were $5^{\prime}$-ATGTGGTCAATGGTCAGCCTTTTTCA-3' (forward) and 5'-TGCCATCACTGCCAACATTCACTCTA-3' (reverse). Real-time quantitative PCR of islet samples or MIN6 $\beta$ cells was performed as previously described (11). The primers used for real-time PCR for mouse obr were 5'-GCTCTTCTGATGTATTTGGAAATC-3' (forward) and 5'-ACCTGATATTGAAGCGGAAATGG-3' (reverse); for mouse SOCS-3 were 5'-GGGGGAGGCAGGAGGTGATGG-3' (forward) and 5'-GGGCGGGCTGGAGGTGGATT-3' (reverse); and for mouse insulin I were $5^{\prime}$-CCTGTTGGTGCACTTCCTA-3' (forward) and 5'-TCTGAAGGTCCCCGGGGCT-3' (reverse).
In situ bybridization for $\mathrm{ObRb}$. Mouse brain was dissected and pretreated as described previously (40). For in situ hybridization for ObR mRNA in the mouse brain, we followed the protocol as previously described (41) by using a probe specific for ObRb (42).

Measurement of insulin secretion and $\mathrm{Ca}^{2+}$ flux in islets. Mouse islets were isolated as described above, and single size-matched islets were incubated in different concentrations of glucose with or without mouse recombinant leptin (Sigma-Aldrich) as indicated and assayed for insulin secretion and intracellular $\mathrm{Ca}^{2+}$ concentration as described previously (10).

$\beta$ Cell mass, size, and immunohistochemistry. Mice were anesthetized, and pancreas was rapidly dissected, weighed, fixed in Bouin's solution, sectioned, and stained; and $\beta$ cell mass was calculated by morphometric analysis as described previously $(23,24)$. Antibodies used for the immunofluorescence staining were as follows; insulin: guinea pig antihuman insulin (Linco) and Texas red-conjugated donkey anti-guinea pig IgG (Jackson ImmunoResearch Laboratories Inc.); glucagon: rabbit antiglucagon antibody (Zymed) and FITC-conjugated goat anti-rabbit antibody (Zymed); $\beta$-catenin: mouse anti- $\beta$-catenin antibody (BD Biosciences) and Cy2-conjugated goat anti-mouse IgG (Jackson ImmunoResearch Laboratories Inc.); Glut-2: rabbit anti-Glut-2 antibody (Alpha Diagnostic International) and Cy2-conjugated anti-rabbit IgG (Jackson ImmunoResearch Laboratories Inc.); BrdU: mouse monoclonal BrdU antibody (Dako) and Cy2-conjugated goat anti-mouse IgG (Jackson ImmunoResearch Laboratories Inc.); cleaved caspase-3: rabbit polyclonal anti-cleaved caspase-3 (R\&D Systems) and Texas red-conjugated donkey anti-rabbit antibody (Jackson ImmunoResearch Laboratories Inc.). $\beta$ Cell and islet size were analyzed using ImageJ software (NIH; http://rsb.info.nih.gov/ij/). DAPI (Sigma-Aldrich) was used for nuclear staining.

Cell culture and siRNA transfection. MIN6 $\beta$ cells were incubated at $37^{\circ} \mathrm{C}$ and $5 \% \mathrm{CO}_{2}$ in DMEM supplemented with $15 \% \mathrm{FBS}$ and penicillin and streptomycin. Predesigned siRNA for mouse ObR (Ambion) was transfected in MIN6 $\beta$ cells using Lipofectamine 2000 reagent (Invitrogen) according to the manufacturer's instructions. Briefly, $100 \mathrm{nM}$ of siRNA was mixed with Lipofectamine 2000 in Opti-MEM I medium and incubated at room temperature for 20 minutes. MIN6 $\beta$ cells were washed and incubated in antibiotic-free DMEM with $15 \%$ FBS before transfection. The siRNA-Lipofectamine mixture was added to the cells and incubated for approximately 8 hours at $37^{\circ} \mathrm{C}$ and $5 \% \mathrm{CO}_{2}$ before being replaced with normal culture medium. The cells were harvested for protein or RNA extraction 48 hours after transfection.

Western blotting. Protein samples extracted from isolated islets or cell lysates were subjected to SDS-PAGE and immunoblotting (23). Antibodies against Akt, phospho-Akt (Ser473), p70S6K, phospho-p70S6K (Thr389), FoxO1, phospho-FoxO1 (Ser256), phospho-PTEN (Ser380/Thr382/383), PTEN, and phospho-Stat3 (Tyr705) were from Cell Signaling Technology. Anti- $\alpha$-tubulin antibody was from Abcam. Anti-Glut-2, -Stat3, and -Jak2 antibodies were from Santa Cruz Biotechnology Inc. Anti-phospho-Jak2 (Tyr1007/1008) was from Biosource. HRP-conjugated goat anti-rabbit IgG and goat anti-mouse IgG were from Santa Cruz Biotechnology Inc. Intensity of the bands was quantified using ImageJ software.

Statistics. All data are presented here as mean \pm SEM and were analyzed using an unpaired 2-tailed Student's $t$ test or analysis of variance (ANOVA) as appropriate. A $P$ value less than 0.05 was considered significant.

\section{Acknowledgments}

The authors thank K.C. Hayes (Brandeis University) for assistance with care of mouse colonies, Jeffrey Friedman (Rockefeller University) for the ObRlox mice, Douglas Melton (Harvard University) for the Pdx-Cre mice, and Kendra Reid and Ryo Suzuki for technical assistance. This work was supported by NIH grants R01 DK67536 (to R.N. Kulkarni) and R21 DK75766 (to R.N. 
Kulkarni); American Diabetes Association Research Grants 7-07-RA-84 (to R.N. Kulkarni) and R37 DK46960 (to R.T. Kennedy); and Joslin DERC grant P30 DK36836 (Specialized Assay and Advanced Microscopy Cores). T. Morioka is supported by the Lilly International Fellowship Program for 2005-2006 and the Noguchi Medical Research Institute.

1. Myers, M.G., Jr. 2004. Leptin receptor signaling and the regulation of mammalian physiology. Recent Prog. Horm. Res. 59:287-304

2. Kieffer, T.J., and Habener, J.F. 2000. The adipoinsular axis: effects of leptin on pancreatic beta-cells. Am. J. Physiol. Endocrinol. Metab. 278:E1-E14.

3. Kulkarni, R.N., et al. 1997. Leptin rapidly suppresses insulin release from insulinoma cells, rat and human islets and, in vivo, in mice. J. Clin. Invest. 100:2729-2736.

4. Poitout, V., Rouault, C., Guerre-Millo, M., and Reach, G. 1998. Does leptin regulate insulin secretion? Diabetes Metab. 24:321-326.

5. Seufert, J., Kieffer, T.J., and Habener, J.F. 1999. Leptin inhibits insulin gene transcription and reverses hyperinsulinemia in leptin-deficient ob/ob mice. Proc. Natl. Acad. Sci. U. S. A. 96:674-679.

6. Lee, G.H., et al. 1996. Abnormal splicing of the leptin receptor in diabetic mice. Nature. 379:632-635.

7. Baetens, D., et al. 1978. Alteration of islet cell populations in spontaneously diabetic mice. Diabetes. 27:1-7

8. Kulkarni, R.N., et al. 1999. Tissue-specific knockout of the insulin receptor in pancreatic beta cells creates an insulin secretory defect similar to that in type 2 diabetes. Cell. 96:329-339.

9. Kulkarni, R.N., et al. 2002. beta-cell-specific deletion of the Igf1 receptor leads to hyperinsulinemia and glucose intolerance but does not alter beta-cell mass. Nat. Genet. 31:111-115.

10. Ueki, K., et al. 2006. Total insulin and IGF-I resistance in pancreatic beta cells causes overt diabetes. Nat. Genet. 38:583-588.

11. Okada, T., et al. 2007. Insulin receptors in \{beta\}cells are critical for islet compensatory growth response to insulin resistance. Proc. Natl. Acad. Sci. U. S. A. 104:8977-8982.

12. Da Silva Xavier, G., Qian, Q., Cullen, P.J., and Rutter, G.A. 2004. Distinct roles for insulin and insulin-like growth factor-1 receptors in pancreatic beta-cell glucose sensing revealed by RNA silencing. Biochem. J. 377:149-158.

13. Hashimoto, N., et al. 2006. Ablation of PDK1 in pancreatic beta cells induces diabetes as a result of loss of beta cell mass. Nat. Genet. 38:589-593.

14. Withers, D.J., et al. 1998. Disruption of IRS-2 causes type 2 diabetes in mice. Nature. 391:900-904.

15. Kubota, N., et al. 2000. Disruption of insulin receptor substrate 2 causes type 2 diabetes because of liver insulin resistance and lack of compensatory beta-cell hyperplasia. Diabetes. 49:1880-1889.

16. Tuttle, R.L., et al. 2001. Regulation of pancreatic
Received for publication November 10, 2006, and accepted in revised form July 6, 2007.

Address correspondence to: Rohit N. Kulkarni, Room 602, One Joslin Place, Boston, Massachusetts 02215, USA. Phone: (617) 713-3460; Fax: (617) 713-3476; E-mail: Rohit.Kulkarni@joslin.harvard.edu. beta-cell growth and survival by the serine/threonine protein kinase Akt1/PKBalpha. Nat. Med. 7:1133-1137.

17. Bernal-Mizrachi, E., Wen, W., Stahlhut, S., Welling, C.M., and Permutt, M.A. 2001. Islet $\beta$ cell expression of constitutively active $\mathrm{Akt} 1 / \mathrm{PKB} \alpha$ induces striking hypertrophy, hyperplasia, and hyperinsulinemia. J. Clin. Invest. 108:1631-1638. doi:10.1172/ JCI200113785.

18. Pende, M., et al. 2000. Hypoinsulinaemia, glucose intolerance and diminished beta-cell size in S6K1deficient mice. Nature. 408:994-997.

19. Nakae, J., et al. 2002. Regulation of insulin action and pancreatic beta-cell function by mutated alleles of the gene encoding forkhead transcription factor Foxo1. Nat. Genet. 32:245-253.

20. Cohen, P., et al. 2001. Selective deletion of leptin receptor in neurons leads to obesity. J. Clin. Invest. 108:1113-1121. doi:10.1172/JCI200113914

21. Lammert, E., et al. 2003. Role of VEGF-A in vascularization of pancreatic islets. Curr. Biol. 13:1070-1074.

22. Islam, M.S., Morton, N.M., Hansson, A., and Emilsson, V. 1997. Rat insulinoma-derived pancreatic beta-cells express a functional leptin receptor that mediates a proliferative response. Biochem. Biophys. Res. Commun. 238:851-855.

23. Kulkarni, R.N., et al. 2003. Impact of genetic background on development of hyperinsulinemia and diabetes in insulin receptor/insulin receptor substrate-1 double heterozygous mice. Diabetes. 52:1528-1534.

24. Michael, M.D., et al. 2000. Loss of insulin signaling in hepatocytes leads to severe insulin resistance and progressive hepatic dysfunction. Mol. Cell. 6:87-97.

25. Ning, K., et al. 2006. A novel leptin signalling pathway via PTEN inhibition in hypothalamic cell lines and pancreatic beta-cells. EMBO J. 25:2377-2387.

26. Ueki, K., Kondo, T., and Kahn, C.R. 2004. Suppressor of cytokine signaling 1 (SOCS-1) and SOCS-3 cause insulin resistance through inhibition of tyrosine phosphorylation of insulin receptor substrate proteins by discrete mechanisms. Mol. Cell. Biol. 24:5434-5446

27. Laubner, K., et al. 2005. Inhibition of preproinsulin gene expression by leptin induction of suppressor of cytokine signaling 3 in pancreatic beta-cells. Diabetes. 54:3410-3417.

28. Unger, R.H., and Zhou, Y.T. 2001. Lipotoxicity of beta-cells in obesity and in other causes of fatty acid spillover. Diabetes. 50(Suppl. 1):S118-S121.

29. Poitout, V., and Robertson, R.P. 2002. Minireview: secondary beta-cell failure in type 2 diabetes--a convergence of glucotoxicity and lipotoxicity. Endocrinology. 143:339-342.

30. Unger, R.H., Zhou, Y.T., and Orci, L. 1999. Regulation of fatty acid homeostasis in cells: novel role of leptin. Proc. Natl. Acad. Sci. U. S. A. 96:2327-2332.

31. Covey, S.D., et al. 2006. The pancreatic beta cell is a key site for mediating the effects of leptin on glucose homeostasis. Cell Metab. 4:291-302.

32. Berti, L., Kellerer, M., Capp, E., and Haring, H.U. 1997. Leptin stimulates glucose transport and glycogen synthesis in C2C12 myotubes: evidence for a P13-kinase mediated effect. Diabetologia. 40:606-609.

33. Szanto, I., and Kahn, C.R. 2000. Selective interaction between leptin and insulin signaling pathways in a hepatic cell line. Proc. Natl. Acad. Sci. U. S. A. 97:2355-2360.

34. Zhao, A.Z., et al. 2000. Leptin induces insulin-like signaling that antagonizes cAMP elevation by glucagon in hepatocytes. J. Biol. Chem. 275:11348-11354.

35. Billington, C.K., et al. 2005. Cooperative regulation of p70S6 kinase by receptor tyrosine kinases and G protein-coupled receptors augments airway smooth muscle growth. Biochemistry. 44:14595-14605.

36. Jetton, T.L., et al. 2005. Mechanisms of compensatory beta-cell growth in insulin-resistant rats: roles of Akt kinase. Diabetes. 54:2294-2304.

37. Uchida, T., et al. 2005. Deletion of Cdkn1b ameliorates hyperglycemia by maintaining compensatory hyperinsulinemia in diabetic mice. Nat. Med. 11:175-182.

38. de Luca, C., et al. 2005. Complete rescue of obesity, diabetes, and infertility in $d b / d b$ mice by neuron-specific LEPR-B transgenes. J. Clin. Invest. 115:3484-3493. doi:10.1172/JCI24059.

39. Ludwig, D.S., et al. 2001. Melanin-concentrating hormone overexpression in transgenic mice leads to obesity and insulin resistance. J. Clin. Invest. 107:379-386.

40. Zigman, J.M., Jones, J.E., Lee, C.E., Saper, C.B., and Elmquist, J.K. 2006. Expression of ghrelin receptor mRNA in the rat and the mouse brain. J. Comp. Newrol. 494:528-548.

41. Elmquist, J.K., Bjorbaek, C., Ahima, R.S., Flier, J.S., and Saper, C.B. 1998. Distributions of leptin receptor mRNA isoforms in the rat brain. J. Comp. Neurol. 395:535-547.

42. Bjorbaek, C., Uotani, S., da Silva, B., and Flier, J.S. 1997. Divergent signaling capacities of the long and short isoforms of the leptin receptor. J. Biol. Chem. 272:32686-32695. 